\title{
Failure rate analysis for time-sensitive networking
}

\author{
Jinpeng $\mathrm{Xu}^{1, \mathrm{a}}$, Feng $\mathrm{Luo}^{1, \mathrm{~b}}$ and Hailun Shao ${ }^{1, \mathrm{c}}$ \\ ${ }^{1}$ Clean Energy Automotive Engineering Center, School of Automotive Studies, Tongji University, \\ Shanghai 201804, China \\ a1310790@tongji.edu.cn, ${ }^{b}$ luo feng@tongji.edu.cn, ${ }^{c} 1434494 @$ tongji.edu.cn
}

Keywords: Ethernet failure rate, Warshall algorithm, flexible position, P802.1CB.

Abstract. With the increasing complexity of automotive network, Ethernet is gradually used for internal communication. In order to satisfy the requirements of vehicle communication, the network needs to have the characteristic of seamless redundancy. IEEE P802.1CB is one of the TSN (Time-Sensitive Networking) active projects, which provides seamless redundancy character for Ethernet network by frame replication and elimination at flexible position to improve reliability.

For a vehicular network, the redundant features lead to cost increase. So it is required to fully understand the influence of network topology on failure rate. However, flexible position means there is no common failure calculation method. This paper introduces one way to calculate failure rate for a given network topology. Calculation results show the frame failure rate between any nodes in the network to help network designers know the effect for redundancy link.

\section{Introduction}

With the increasing complexity of automotive network, traditional Controller Area Network (CAN) cannot satisfy internal communication bandwidth requirements such as enhanced safety and entertainment solutions [1]. So Ethernet is gradually used for internal communication.

Different from CAN bus, automotive Ethernet with BroadR-Reach technology is point-to-point technique, which means several switches are needed when frames are delivered between end stations. For traditional Ethernet network, if one switch fails, the frames will be lost, and several seconds are needed to find a new way for frame delivery, which is unacceptable for vehicle critical communication. In order to provide the specifications that allow time-synchronized low latency streaming services through 802 networks, IEEE 802.1 Time-Sensitive Networking Task Group [2] is founded to satisfy the harsh environment communication requirements.

P802.1CB [3] is one of the TSN (Time-Sensitive Networking) active projects, which provides seamless redundancy character for Ethernet network by frame replication and elimination to improve reliability [4]. Compared with Parallel Redundancy Protocol (PRP) and High-availability Seamless Redundancy (HSR) [5], P802.1CB does not restrict network topology to independent part (PRP) or ring (HSR). So the question comes with flexibility of seamless redundancy is how to evaluate the benefit of redundant topologies.

This paper introduces one way to calculate failure rate for a given network topology. Calculation results show the frame failure rate between any nodes in the network, so network designers can check if the failure rates of all nodes meets their requirements and know the improvement of failure rate from old topologies.

The rest of this paper is organized as follows: in Section 2, the standard P802.1CB seamless redundancy protocol is briefly introduced. Next, Section 3 describes how to calculate failure rate with reachable matrixes. In Section 4, the failure rate for a given topologies is calculated with MATLAB to verify the results. Finally, conclusions and suggestions for future work are provided in Section 5.

\section{Seamless redundancy}

Highly seamless communication with fault tolerance is one of the key requirements for Ethernet-based, mission-critical and real-time systems, such as substation automation system (SAS) networks and other industrial Ethernet networks [6]. Since traditional Ethernet does not provide such 
capability, some fault-tolerant Ethernet protocol have been developed [5]. The parallel redundancy protocol (PRP) and the high-availability seamless redundancy (HSR) protocol, standardized as IEC62439-3, are provided for seamless communication. But these protocol are restricted to ring or independent part as follows:
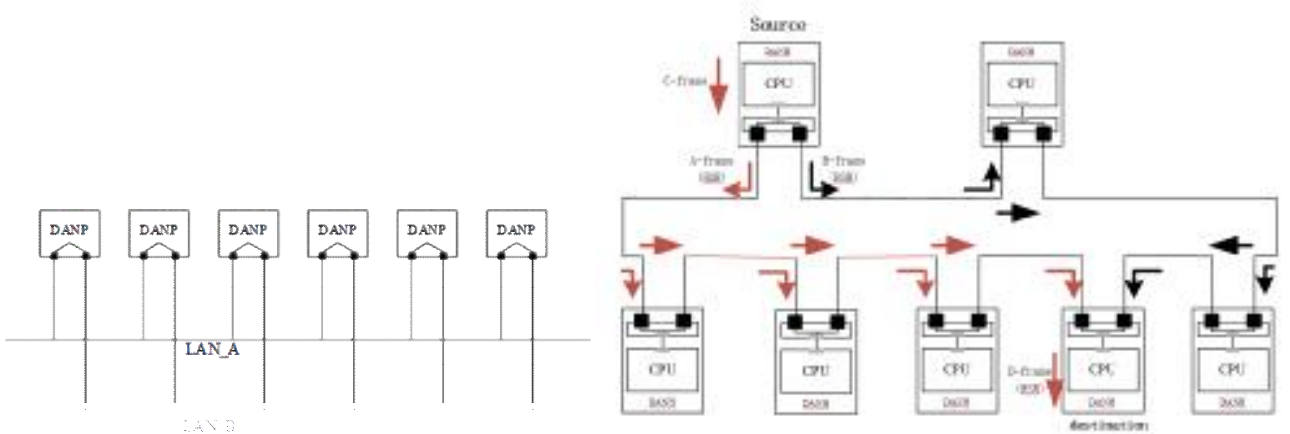

Figure 1. Topologies of PRP and HSR

P802.1CB provides 802-compatible solutions for fault tolerance without failover. Apart from seamless redundancy, flexible position is another character, which means this stander will work on all LAN topologies.

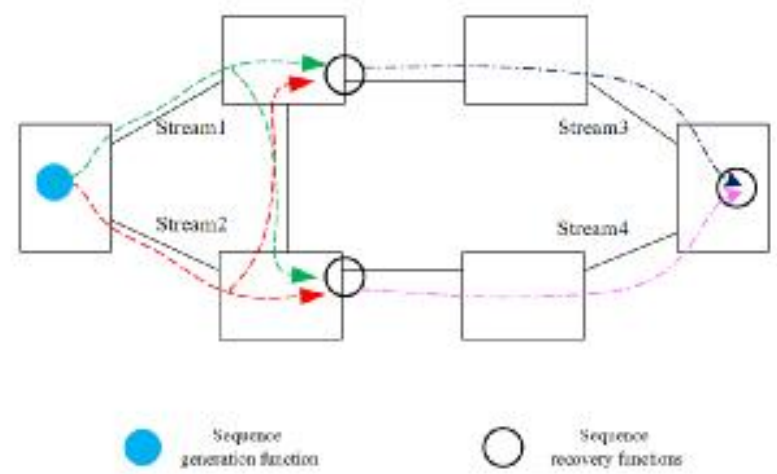

Figure 2. Topologies of P802.1CB

Fig. 2 illustrates an example; a sequence number is generated and encoded into each packet in the leftmost box. Sequence recovery functions eliminate duplicate packets, and the non-duplicate packets are copied as a new Member Stream (with sequence numbers unchanged) at two intermediate points. The final two Member Streams are brought together and the duplicates are eliminated at the destination at right. This configuration protects against all 7 possible one-link failures, and against 16 of 21 possible two-link failures [4].

\section{Failure rate calculation}

\section{Network description}

In order to describe network topology, $\mathrm{V}$ is taken as the set of nodes (a node may be a switch or end station), and $\mathrm{E}$ as the set of lines (full duplex Ethernet link), then $\mathrm{G}=(\mathrm{V}, \mathrm{E})$ is described as the network topology. As the fig. 3 shows, $V=\{1,2,3,4\}, E=\{\{1,2\},\{2,3\},\{2,4\}\}$.

Failure may occur on any lines or any node. For a real network, frames may be lost when failure occurs such as link failure, connector failure and node failure. However, from the view of calculation, the reason for frame lost is not important. Only two types of failure are used. As fig. 3 shows below, line failure means frames fail to pass one link and node failure means node cannot deliver frame to other node. 

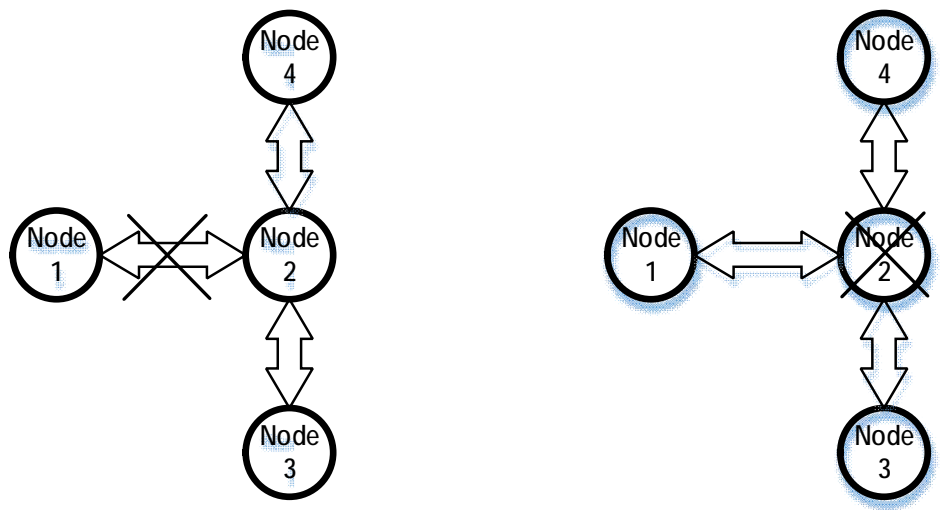

Figure 3. Line failure and node failure

When the network is working, different nodes or lines may have different failure rates. For example, the node works under environment of high electromagnetic interference is more likely to fail compared with regular node. Assume failure rate for node $\mathrm{n}$ is $\mathrm{p}_{\mathrm{n}}$, and failure rate for line between node $\mathrm{k}$ and $\mathrm{j}$ is $\mathrm{p}_{\mathrm{kj}}$, then $\mathrm{V}$ and $\mathrm{E}$ can be extended to $\mathrm{V} 1=\left\{\left\{1, \mathrm{p}_{1}\right\},\left\{2, \mathrm{p}_{2}\right\},\left\{3, \mathrm{p}_{3}\right\},\left\{4, \mathrm{p}_{4}\right\}\right\}$ and $\mathrm{E} 1=$ $\left\{\left\{1,2, \mathrm{p}_{12}\right\},\left\{2,3, \mathrm{p}_{23}\right\},\left\{2,4, \mathrm{p}_{24}\right\}\right\}$.

\section{Network failure rate calculation}

In order to get the failure rate for any given network topology, the simplest way is to enumerate all possible failure states. For each failure state, it is easy to calculate the probability of occurrence. Take the failure state in the left of fig. 3 for example, occurrence $\mathrm{P}=\left(1-\mathrm{p}_{1}\right) \times\left(1-\mathrm{p}_{2}\right) \times\left(1-\mathrm{p}_{3}\right) \times\left(1-\mathrm{p}_{4}\right) \times$ $\mathrm{p}_{12} \times\left(1-\mathrm{p}_{23}\right) \times\left(1-\mathrm{p}_{24}\right)$. At the same time, new set $\mathrm{V} \_\mathrm{sub}=\{1,2,3,4\}$ and $\mathrm{E} \_$sub $=\{\{2,3\},\{2,4\}\}$ can be used to describe this failed network topology. V_sub and E_sub can be translated to adjacency matrix A.

$$
\mathrm{A}=\left[\begin{array}{llll}
1 & 0 & 0 & 0 \\
0 & 1 & 1 & 1 \\
0 & 1 & 1 & 0 \\
0 & 1 & 0 & 1
\end{array}\right] \mathrm{A}^{2}=\left[\begin{array}{llll}
1 & 0 & 0 & 0 \\
0 & 3 & 2 & 2 \\
0 & 2 & 2 & 1 \\
0 & 2 & 1 & 2
\end{array}\right] \quad \mathrm{A}^{3}=\left[\begin{array}{llll}
1 & 0 & 0 & 0 \\
0 & 7 & 5 & 5 \\
0 & 5 & 4 & 3 \\
0 & 5 & 3 & 4
\end{array}\right]
$$

$A$ is an $n \times n$ matrix ( $n$ is number of nodes), if there are $k$ lines between node $i$ and node $j$, then $a_{i j}=a_{j i}=k$. Matrix $A, A^{2}, A^{3}$ show the reachability between nodes. Node $i$ and node $j$ is reachable as long as $a_{i j}$ in any of three matrixes is not zero. The failure rate between node $i$ and node $j$ should be the sum of all $\mathrm{P}$ which node $\mathrm{i}$ and node $\mathrm{j}$ are unreachable.

\section{Calculation acceleration}

With the increasing number of nodes, the algorithm is significantly increased. For a network with $\mathrm{n}$ nodes and $\mathrm{k}$ lines, there are $2^{(\mathrm{n}+\mathrm{k})}$ possibilities of failure states. For each state, $\mathrm{n}-2$ matrix multiplications are needed. This is not an easy job thus some methods are used to reduce calculation.

(1)Network simplification

Sometimes there are simple nodes in the network. As fig. 4 shows, these nodes are connected as a chain. If any line or node fails, this chain is break. So this chain can be simplified to one node, $\mathrm{p}_{1 \_\mathrm{n}}=$ $1-\left(1-\mathrm{p}_{1}\right) \times\left(1-\mathrm{p}_{2}\right) \times \ldots \times\left(1-\mathrm{p}_{\mathrm{n}}\right) \times\left(1-\mathrm{p}_{12}\right) \times \ldots \times\left(1-\mathrm{p}_{(\mathrm{n}-1) \mathrm{n}}\right)$. Then the number of enumeration is reduced.

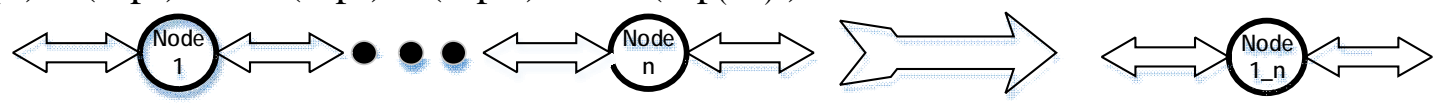

(2)Warshall algorithm

Figure 4. Network simplification

For each failure state, Warshall algorithm can be used to reduce the amount of calculation and run as the following steps:

(a) Get matrix A from V_sub and E_sub;

(b) Set $i=1$;

(c) For $\mathrm{j}=1: \mathrm{n}$, if $A(j, i)==1$, then for $\mathrm{k}=1: \mathrm{n}, \mathrm{A}(\mathrm{j}, \mathrm{k})=\mathrm{A}(\mathrm{j}, \mathrm{k}) \mid \mathrm{A}(\mathrm{i}, \mathrm{k})$;

(d) $\mathrm{i}=\mathrm{i}+1$;

(e) if $\mathrm{i}<=\mathrm{n}$, then turn to step 3, otherwise stop; 
For step 3, after loops for all $\mathrm{j}, \mathrm{A}(\mathrm{m}, \mathrm{n})=\mathrm{A}(\mathrm{m}, \mathrm{n}) \mid(\mathrm{A}(\mathrm{m}, \mathrm{i}) \& \mathrm{~A}(\mathrm{i}, \mathrm{n}))$. In addition, full duplex Ethernet means matrix $\mathrm{A}$ is a symmetric matrix. Thus $\mathrm{A}(\mathrm{m}, \mathrm{n})|(\mathrm{A}(\mathrm{m}, \mathrm{i}) \& \mathrm{~A}(\mathrm{i}, \mathrm{n}))==\mathrm{A}(\mathrm{n}, \mathrm{m})|(\mathrm{A}(\mathrm{i}$, m) \& $A(n, i))==A(n, m)$. After step 3, the symmetric character is not changed. Therefore, step (3) can be changed to reduce algorithm.

For $j=1: n$, if $A(j, i)==1 \& \& i \neq j$, then for $k=j+1: n, A(j, k)=A(j, k) \mid A(i, k), A(k, j)=A(j, k)$;

\section{Algorithm implementation}

The calculation method in the last chapter is easy to implement with MATLAB (Matrix Laboratory). In order to verify the correctness of the calculation process, various network structures can be used to validate the algorithm.

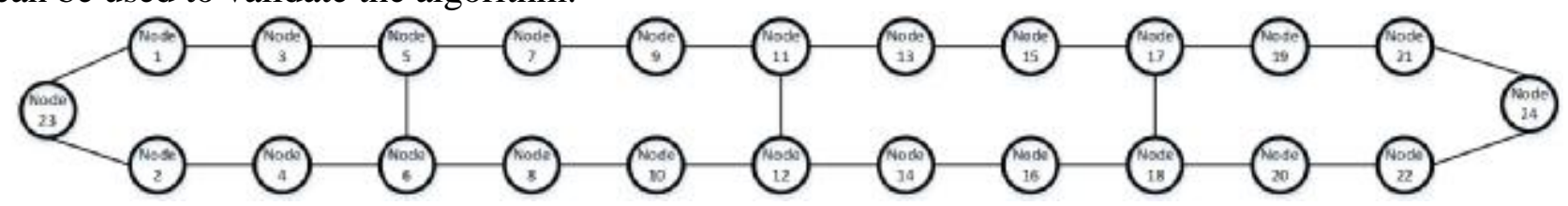

Figure 5. 24 nodes with cross-link

As fig. 5 shows, it is a ring network with three cross-links. According to analysis [7], more cross-link will lead to better network performance. Assume node failure rate is 0.0024 , and then frame failure rate between node 23 and node 24 is $2.71 \mathrm{e}-04$. However, reference [7] introduced only node failure without link failure and lack results for other nodes. In order to calculate the failure rate of other nodes, the method of calculation in this paper can be used.

Assume node failure rate is still 0.0024, and set line failure rate to 0.0001, then V1 and E1 can be generated:

$$
\mathrm{V} 1=\{\{1,0.0024\},\{2,0.0024\} \ldots\{23,0.0024\},\{24,0.0024\}\}
$$

$\mathrm{E} 1=\{\{1,3,0.0001\},\{3,5,0.0001\} \ldots\{24,2,0.0001\},\{24,1,0.0001\}\}$

There are $\mathrm{C}_{24}^{2}=276$ results for the network in fig. 5, as table1 and fig. 6 show below.

Table 1 Network failure rate

\begin{tabular}{|c|c|c|c|c|}
\hline Node x1 & Node x2 & $\begin{array}{c}\text { Failure rate (no } \\
\text { redundancy) }\end{array}$ & Failure rate & $\begin{array}{c}\text { Improvement } \\
\text { factor }\end{array}$ \\
\hline 1 & 2 & 0.00738128735 & 0.00482001498 & 1.5314 \\
\hline 1 & 3 & 0.00489376058 & 0.00479547783 & 1.0205 \\
\hline$\ldots$ & $\ldots$ & $\ldots$ & $\ldots$ & $\ldots$ \\
\hline 5 & 6 & 0.00489376058 & 0.00479425860 & 1.0208 \\
\hline$\ldots$ & $\ldots$ & $\ldots$ & $\ldots$ & $\ldots$ \\
\hline 22 & 24 & 0.00489376058 & 0.00479547783 & 1.0205 \\
\hline 23 & 24 & 0.03191710498 & 0.00508141170 & 6.2811 \\
\hline
\end{tabular}




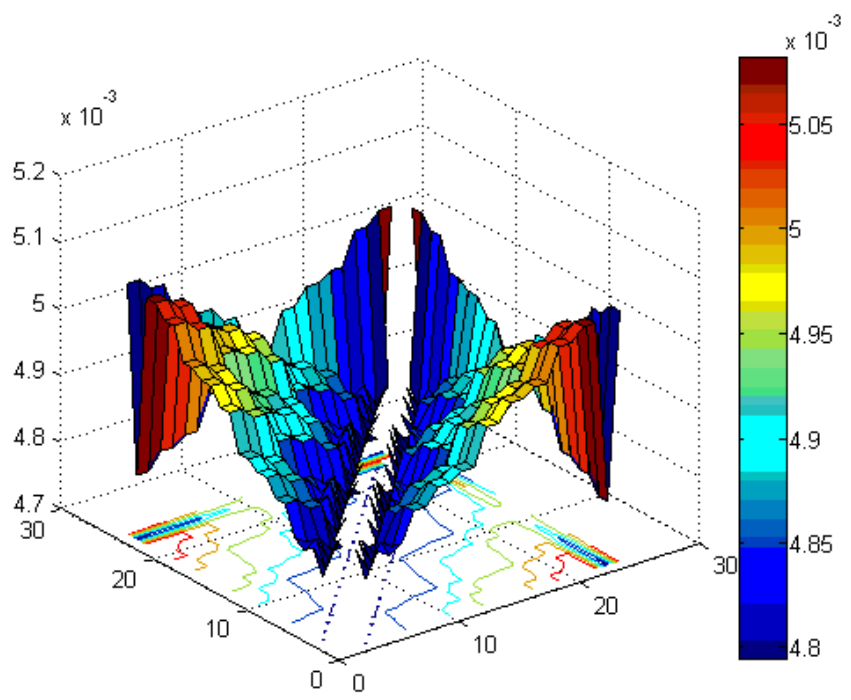

Figure 6. Network failure rate

After the data is sorted, the most likely failure of frame transmission occurs between nodes 23 and 24 with failure rate 0.0050814117 . The least likely failure is 0.0047942586 between nodes 5 and 6 or between nodes 17 and 18 .

\section{Conclusions}

This paper demonstrates failure rate calculation process of seamless redundancy network. The definition of input data, calculation principle and optimization method are introduced. Finally, an example network is analyzed with MATLAB and the results show the failure rate for frame delivery. It also show the benefit of redundancy of network. For network designers, these results can be used to analyze the reliability of the network. By comparing the calculation results of different networks, designers could clearly understand the influence of each connection, and it is convenient to remove the over redundant connection to ensure the economy of the design.

\section{Acknowledgements}

This work was financially supported by Shanghai Automotive Industry Science and Technology Development Foundation (1515).

\section{References}

[1] Hank Peter, Müller Steffen, etc. Automotive Ethernet: In-vehicle Networking and Smart Mobility: Proceedings of the Conference on design, automation and test in europe, 2013; 1-2.

[2] IEEE Std, Time-Sensitive Networking Task Group. [Online]. Available: http://www.ieee802.org/1/pages/tsn.html

[3] IEEE Std, Frame Replication and Elimination for Reliability. [Online]. Available: http://www.ieee802.org/1/pages/802.1cb.html

[4] IEEE Std, Draft Standard for Local and metropolitan area networks -Frame Replication and $\begin{array}{llll}\text { Elimination for Reliability. } & \text { [Online]. Available: }\end{array}$ http://www.ieee802.org/1/files/private/cb-drafts/d2/802-1CB-d2-3.pdf

[5] IEC 65C, "Industrial communication networks - High availability automation networks - Part 3: Parallel Redundancy Protocol (PRP) and High-availability Seamless Redundancy (HSR)," International Electrotechnical Commission, Geneva, Switzerland, IEC 62439-3, Jul. 2012. 
[6] Nsaif, S.A.; Rhee, J.-M. DVP: A Novel High-Availability Seamless Redundancy (HSR) Protocol Traffic-Reduction Algorithm for a Substation Automation System Network. Energies 2014, 7, 1792-1810.

[7] Norman Finn; Failure rates and P802.1CB. [Online]. Available: http://www.ieee802.org/1/files/public/docs2013/cb-nfinn-packet-loss-ratio-10-13-v03.pdf 